\title{
SOME SPECTRAL APPROXIMATIONS OF TWO-DIMENSIONAL FOURTH-ORDER PROBLEMS
}

\author{
CHRISTINE BERNARDI, GIUSEPPE COPPOLETTA, AND YVON MADAY
}

\begin{abstract}
This paper deals with the approximation of the biharmonic equation in a square domain with Dirichlet boundary condixions. Two types of discrete problems are presented, the numerical analysis is performed, and estimates for the error between the exact and approximate solutions are given.
\end{abstract}

\section{INTRODUCTION}

We are interested in the spectral discretization of the following biharmonic equation:

$$
\Delta^{2} u=f \quad \text { in } \Omega,
$$

where $\Omega$ is a square domain, when it is provided with Dirichlet boundary conditions, i.e., the values of $u$ and of its normal derivative are prescribed on the whole boundary of the domain. This equation is known as the plate problem. As another important application of our results, we quote the Stokes equations which govern the flow of a stationary incompressible viscous fluid: indeed, the incompressibility condition is equivalent to the existence of a stream function which, in the two-dimensional case, is a solution of problem (1.1). Using this formulation allows for satisfying exactly the incompressibility equation while avoiding the drawback of spurious modes on the pressure (we refer to [5] for a review of spectral methods for the Stokes problem in the primitive variables of velocity and pressure). This leads to a low-cost discretization, since only one scalar unknown is computed, and the velocity can easily be recovered from the stream function by a standard spectral derivation process. The method can be extended to handle the nonlinear terms of the full Navier-Stokes equations in a natural way.

The discretizations which we propose for problem (1.1) are of spectral type, i.e., the approximate solution is sought in a finite-dimensional space of polynomials of high degree. This space seems especially appropriate to treat high-order problems, since it consists of infinitely differentiable functions, in contrast to finite element discrete spaces (this property has already been used in the case of Fourier series, see, e.g., $[16,11])$. We propose here two kinds of spectral discretizations. The first method is of collocation type, which means that equation (1.1) is enforced in a finite number of points, called collocation points,

Received by the editor October 8, 1990 and, in revised form, July 22, 1991.

1991 Mathematics Subject Classification. Primary 65N35; Secondary 65D30. 
which form a tensorized grid. In each direction, the coordinates of these points are chosen as the nodes of a one-dimensional quadrature formula, so that a variational formulation of the discrete problem can be derived in a natural way and the Lagrange interpolation operator in the points of the grid has optimal approximation properties. Consequently, the nodes are the zeros of one of the derivatives of a Legendre polynomial. In order to use the most accurate quadrature formula, while taking the boundary conditions into account, we construct the collocation nodes from a generalized Gauss-type formula which was studied in detail in [2]. The second method is no longer a pure collocation method, but still involves quadrature formulas of the same type. The numerical analysis of the discrete problem proves that the approximation has an infinite order of accuracy for smooth solutions. Numerical tests, performed by Koutchmy [12], are in complete agreement with the results of this paper.

Only for the sake of brevity, the analysis will be presented for two-dimensional domains and homogeneous boundary conditions: the extension to the case of a cube is straightforward, the complete analysis for inhomogeneous conditions is given in [1]. Modified discrete problems can be derived by replacing the Legendre polynomials by Jacobi ones in the definition of the quadrature formulas; we also refer to [1, Appendix] for details.

The outline of the paper is as follows. Some basic notation concerning the continuous problem and the polynomial approximation are given in $\S 2$. In $\S 3$, we propose a collocation method and give a bound for the error between the exact and discrete solutions. However, this error is not of optimal order; that is why we propose in $\S 4$ another discretization which is no longer of collocation type but leads to optimal error estimates. Conclusions are given in $\S 5$.

\section{NotATION AND BASIC RESULTS}

The domain $\Omega$ is the open square $\Lambda^{2}$, where $\Lambda$ stands for the interval $(-1,1)$. The generic point in $\Omega$ is written $\mathbf{x}=(x, y)$. We denote by $\Gamma_{J}$, $J=1,2,3,4$, the four edges of the square, starting from the west and turning counterclockwise. The extremities of the edge $\Gamma_{J}$ are the corners $\mathbf{a}_{J-1}$ and $\mathbf{a}_{J}$ (with the conventional notation $\mathbf{a}_{0}=\mathbf{a}_{4}$ ), while $\mathbf{n}_{J}$ stands for the unit outward normal to $\Gamma_{J}$ (with also $\mathbf{n}_{5}=\mathbf{n}_{1}$ ) and $\boldsymbol{\tau}_{J}$ for the unit vector orthogonal to $\mathbf{n}_{J}$, turning counterclockwise.

\subsection{The continuous problem.}

Notation. For any bounded domain $\mathscr{O}$ of $\mathbb{R}^{d}$ with a Lipschitz-continuous boundary, we associate with each positive integer $m$ the Sobolev space $H^{m}(\mathscr{O})$ of all functions in $L^{2}(\mathscr{O})$ such that their partial derivatives of order $\leq m$ belong to $L^{2}(\mathscr{O})$. It is provided with the usual norm $\|\cdot\|_{m, \mathscr{O}}$. The closure of the space of infinitely differentiable functions with a compact support in $\mathscr{O}$ is denoted by $H_{0}^{m}(\mathscr{O})$, and its dual space by $H^{-m}(\mathscr{O})$; the duality pairing is denoted by the symbol $\langle\cdot, \cdot\rangle$. We recall that the usual seminorm $|\cdot|_{m, \mathscr{O}}$ is a norm on $H_{0}^{m}(\mathscr{O})$, equivalent to the norm $\|\cdot\|_{m, \mathscr{O}}$, and we denote by $\|\cdot\|_{-m, \mathscr{O}}$ the corresponding norm on $H^{-m}(\mathscr{O})$. For any nonnegative real number $s$, the space $H^{s}(\mathscr{O})$ is defined by Hilbert interpolation of index $[s]+1-s$ between $H^{[s]+1}(\mathscr{O})$ and $H^{[s]}(\mathscr{O})$ (where [s] stands for the integral part of $s$ ); it is provided with the norm $\|\cdot\|_{s, \mathscr{O}}$. We refer to [13] for properties of Sobolev spaces. 
For given data $f$, we are interested in the approximation of the following fourth-order equation:

$$
\Delta^{2} u=f \quad \text { in } \Omega,
$$

provided with the Dirichlet boundary conditions

$$
u=\frac{\partial u}{\partial n_{J}}=0 \quad \text { on } \Gamma_{J}, J=1,2,3,4 .
$$

To write the variational formulation of problem $(2.1)-(2.2)$, we define on $H^{2}(\Omega) \times H^{2}(\Omega)$ the bilinear form

$$
a(u, v)=\int_{\Omega} \Delta u \Delta v d \mathbf{x}
$$

Thus, problem (2.1)-(2.2) is clearly equivalent to the following one:

$$
\text { find } u \text { in } H_{0}^{2}(\Omega) \text { such that }
$$

$$
\forall v \in H_{0}^{2}(\Omega), \quad a(u, v)=\langle f, v\rangle .
$$

Since the form $a(\cdot, \cdot)$ is elliptic on $H_{0}^{2}(\Omega)$, for any $f$ in $H^{-2}(\Omega)$, problem (2.1)-(2.2) admits a unique solution in $H^{2}(\Omega)$. Moreover, this solution satisfies

$$
\|u\|_{2, \Omega} \leq c\|f\|_{-2, \Omega} \text {. }
$$

\subsection{Polynomial approximation results.}

Notation. For any interval or square domain $\mathscr{O}$, and for any nonnegative integer $n, \mathbf{P}_{n}(\mathscr{O})$ stands for the space of all polynomials on $\mathscr{O}$ with degree $\leq n$ with respect to each variable. We denote by $\mathbf{P}_{n}^{0}(\mathscr{O})$ the subspace $\mathbf{P}_{n}(\mathscr{O}) \cap H_{0}^{1}(\mathscr{O})$ and by $\mathbf{P}_{n}^{00}(\mathscr{O})$ the subspace $\mathbf{P}_{n}(\mathscr{O}) \cap H_{0}^{2}(\mathscr{O})$.

A basis of the space of all polynomials on $\Lambda$ is given by the family of Legendre polynomials $\left(L_{n}\right)_{n \geq 0}$ : the polynomial $L_{n}, n \geq 0$, is orthogonal to any polynomial $L_{m}, m \neq n$, in $L^{2}(\Lambda)$, it has degree $n$ and satisfies $L_{n}(1)=1$. We recall $[7, \S 1.13]$ some properties which will be of constant use. Each polynomial $L_{n}, n \geq 0$, satisfies the differential equation

$$
\left(\left(1-\zeta^{2}\right) L_{n}^{\prime}\right)^{\prime}(\zeta)+n(n+1) L_{n}(\zeta)=0
$$

and the integral equation

$$
\int L_{n} d \zeta=\frac{1}{2 n+1}\left(L_{n+1}-L_{n-1}\right), \quad n \geq 1,
$$

where $\int L_{n} d \zeta$ stands for the primitive of $L_{n}$ which vanishes at \pm 1 . The sequence of polynomials $\left(L_{n}\right)_{n \geq 0}$ is given by the recursion formula

$$
\left\{\begin{array}{l}
(n+1) L_{n+1}(\zeta)=(2 n+1) \zeta L_{n}(\zeta)-n L_{n-1}(\zeta), \quad n \geq 1, \\
L_{0}(\zeta)=1 \text { and } L_{1}(\zeta)=\zeta .
\end{array}\right.
$$

For a fixed positive integer $M$, we denote by $\Pi_{M}^{2,0}$ the orthogonal projection operator from $H_{0}^{2}(\Omega)$ onto $\mathbf{P}_{M}^{00}(\Omega)$. We recall the following estimate of the approximation error [3], which is valid for any real numbers $r$ and $s$ such that $0 \leq r \leq 2 \leq s$ :

$$
\forall \varphi \in H^{s}(\Omega) \cap H_{0}^{2}(\Omega), \quad\left\|\varphi-\Pi_{M}^{2,0} \varphi\right\|_{r, \Omega} \leq c M^{r-s}\|\varphi\|_{s, \Omega} .
$$


Let us fix two integers $m \geq 0$ and $M \geq m$. We denote by $\xi_{j}^{M, m}, 1 \leq$ $j \leq M-m$, the zeros of the polynomial $d^{m} L_{M} / d \zeta^{m}$ in increasing order. It is proved in $[2, \S 2]$ that there exist positive weights $\rho_{j}^{M, m}, 1 \leq j \leq M-m$, and $\rho_{0}^{M, m, k}, 0 \leq k \leq m-1$, such that the quadrature formula

$$
\begin{aligned}
\int_{-1}^{1} \Phi(\zeta) d \zeta \simeq & \sum_{j=1}^{M-m} \Phi\left(\xi_{j}^{M, m}\right) \rho_{j}^{M, m} \\
& +\sum_{k=0}^{m-1}\left(\frac{d^{k} \Phi}{d \zeta^{k}}(-1)+(-1)^{k} \frac{d^{k} \Phi}{d \zeta^{k}}(1)\right) \rho_{0}^{M, m, k}
\end{aligned}
$$

is exact for all polynomials of $\mathbf{P}_{2 M-1}(\Lambda)$. This formula, which is the cornerstone of the discretization method, is the Gauss-Lobatto formula with end points of multiplicity $m$. It is studied in [2] in a more general case and an efficient way of computing its nodes and weights is described in this paper (see also [9]). In what follows, formula (2.10) will be used for $m$ equal to 1,2 , and 3.

We recall that the points $\xi_{j}^{M, m}, 1 \leq j \leq M-m$, are also the nodes of a plain Gauss quadrature formula for the measure $\left(1-\zeta^{2}\right)^{m} d \zeta$. Consequently, it follows from [14] that, for $m$ equal to 1,2 , or 3 , if $i_{M}^{m}$ denotes the Lagrange interpolation operator at these nodes (with values in $\mathbf{P}_{M-m-1}(\Lambda)$ ), the following estimate holds for any real number $s>\frac{1}{2}$ and for any function $\varphi$ such that $\left(1-\zeta^{2}\right) \varphi$ belongs to $H^{s}(\Lambda)$ :

$$
\left\|\left(1-\zeta^{2}\right)\left(\varphi-i_{M}^{m} \varphi\right)\right\|_{0, \Lambda} \leq c N^{-s}\left\|\left(1-\zeta^{2}\right) \varphi\right\|_{s, \Lambda} .
$$

Then, denoting by $\mathcal{F}_{M}^{m}$ the Lagrange interpolation operator on the grid made of the points $\left(\xi_{j}^{M, m}, \xi_{k}^{M, m}\right), 1 \leq j, k \leq M-m$, we derive the following estimate by a tensorization argument: for any real number $s>1$ and for any function $f$ such that $\left(1-x^{2}\right)\left(1-y^{2}\right) f$ belongs to $H^{s}(\Omega)$,

$$
\left\|\left(1-x^{2}\right)\left(1-y^{2}\right)\left(f-\mathcal{I}_{M}^{m} f\right)\right\|_{0, \Omega} \leq c N^{-s}\left\|\left(1-x^{2}\right)\left(1-y^{2}\right) f\right\|_{s, \Omega} .
$$

\section{THE COLLOCATION METHOD}

This section is divided in two parts: the first contains the statement of the discrete problem, the second its numerical analysis.

3.1. The discrete problem. In order to define the discrete problem, we always assume that the function $f$ is continuous on $\Omega$. We fix an integer $N \geq 4$. We consider the points $\xi_{j}^{N-1,2}, 1 \leq j \leq N-3$, and the weights $\rho_{j}^{N-1,2}$, $1 \leq j \leq N-3, \rho_{0}^{N-1,2,0}$, and $\rho_{0}^{N-1,2,1}$ of $\S 2$, which we denote respectively by $\xi_{j}$ and $\rho_{j}, \rho_{0}$ and $\rho_{0}^{*}$ for the sake of brevity. Then, we set

$$
\Xi_{N}=\left\{\left(\xi_{j}, \xi_{k}\right), 1 \leq j, k \leq N-3\right\} .
$$

We also set

$$
\begin{aligned}
& \Xi_{N 1}=\left\{\left(-1, \xi_{j}\right), 1 \leq j \leq N-3\right\}, \\
& \Xi_{N 2}=\left\{\left(\xi_{j},-1\right), 1 \leq j \leq N-3\right\}, \\
& \Xi_{N 3}=\left\{\left(1, \xi_{j}\right), 1 \leq j \leq N-3\right\}, \\
& \Xi_{N 4}=\left\{\left(\xi_{j}, 1\right), 1 \leq j \leq N-3\right\} .
\end{aligned}
$$


We now formulate our discrete problem: find $u_{N}$ in $\mathbf{P}_{N}(\Omega)$ such that

$$
\Delta^{2} u_{N}(\mathbf{x})=f(\mathbf{x}), \quad \mathbf{x} \in \mathbf{\Xi}_{N}
$$

and

$$
\begin{aligned}
u_{N}(\mathbf{x})= & \frac{\partial u_{N}}{\partial n_{J}}(\mathbf{x})=0, \quad \mathbf{x} \in \Xi_{N J}, \quad J=1,2,3,4, \\
u_{N}\left(\mathbf{a}_{J}\right) & =\frac{\partial u_{N}}{\partial n_{J}}\left(\mathbf{a}_{J}\right)=\frac{\partial u_{N}}{\partial n_{J+1}}\left(\mathbf{a}_{J}\right) \\
& =\frac{\partial^{2} u_{N}}{\partial n_{J} \partial n_{J+1}}\left(\mathbf{a}_{J}\right)=0, \quad J=1,2,3,4 .
\end{aligned}
$$

This problem gives rise to a linear system of $(N+1)^{2}$ equations in $(N+1)^{2}$ unknowns: indeed, $(N+1)^{2}$ is the dimension of $\mathbf{P}_{N}(\Omega)$, and there are $(N-3)^{2}$ equations at the interior of the domain, plus $2(N-3)$ on each edge, plus four at each corner.

In order to write a variational formulation of problem (3.3)-(3.5), we define a bilinear form $(\cdot, \cdot)_{N}$ : for any functions $\varphi$ and $\psi$ with continuous derivatives on $\bar{\Lambda}$, we set

$$
\begin{aligned}
(\varphi, \psi)_{N}=\sum_{j=1}^{N-3} \varphi\left(\xi_{j}\right) \psi\left(\xi_{j}\right) \rho_{j} & +(\varphi(-1) \psi(-1)+\varphi(1) \psi(1)) \rho_{0} \\
& +\left((\varphi \psi)^{\prime}(-1)-(\varphi \psi)^{\prime}(1)\right) \rho_{0}^{*} .
\end{aligned}
$$

Similarly, we define a bilinear form on $\mathscr{C}^{1}(\bar{\Omega}) \times \mathscr{C}^{1}(\bar{\Omega})$ in the following way: the quantity $\langle u, v\rangle_{N}$, which approximates the integral $\int_{\Omega} u v d \mathbf{x}$, is obtained by replacing the integrals with respect to each variable by the discrete formula (2.10) applied to the function $u v$. This is simpler to understand than to write out; note, however, that whenever one of the two functions $u$ and $v$ belongs to $H_{0}^{2}(\Omega)$, the definition reduces to

$$
\langle u, v\rangle_{N}=\sum_{j=1}^{N-3} \sum_{k=1}^{N-3} u\left(\xi_{j}, \xi_{k}\right) v\left(\xi_{j}, \xi_{k}\right) \rho_{j} \rho_{k} .
$$

Next, we set

$$
a_{N}(u, v)=\left\langle\Delta^{2} u, v\right\rangle_{N}
$$

Our first result is stated in the following proposition.

Proposition 3.1. Problem (3.3)-(3.5) is equivalent to the following variational problem:

$$
\begin{aligned}
& \text { find } u_{N} \text { in } \mathbf{P}_{N}^{00}(\Omega) \text { such that } \\
& \quad \forall v_{N} \in \mathbf{P}_{N}^{00}(\Omega), \quad a_{N}\left(u_{N}, v_{N}\right)=\left\langle f, v_{N}\right\rangle_{N} .
\end{aligned}
$$

Proof. First, note that conditions (3.4)-(3.5) are equivalent to the fact that $u_{N}$ belongs to $\mathbf{P}_{N}^{00}(\Omega)$ : indeed, on each edge $\Gamma_{J}, J=1,2,3,4, u_{N}$ is a polynomial of degree $\leq N$, it vanishes in $N-1$ points, and its derivative vanishes at the two extremities. Second, choosing $v_{N}$ equal to the only polynomial in $\mathbf{P}_{N}^{00}(\Omega)$ which is equal to 1 at $\left(\xi_{j}, \xi_{k}\right)$ and vanishes at any other point of $\Xi_{N}$ 
for a fixed pair $(j, k), 1 \leq j, k \leq N-3$, we see that (3.8) implies (3.3). Conversely, since these polynomials form a basis of $\mathbf{P}_{N}^{00}(\Omega)$, (3.3) implies (3.8).

The following subsection is devoted to the numerical analysis of problem (3.8).

3.2. Numerical analysis. First note that, for any polynomials $u_{N}$ and $v_{N}$ in $\mathbf{P}_{N}^{00}(\Omega)$, we have

$$
a_{N}\left(u_{N}, v_{N}\right)=\left\langle\frac{\partial^{4} u_{N}}{\partial x^{4}}, v_{N}\right\rangle_{N}+2\left\langle\frac{\partial^{4} u_{N}}{\partial x^{2} \partial y^{2}}, v_{N}\right\rangle_{N}+\left\langle\frac{\partial^{4} u_{N}}{\partial y^{4}}, v_{N}\right\rangle_{N},
$$

so that the exactness of the quadrature formula (2.10) on $\mathbf{P}_{2 N-3}(\Lambda)$ gives

$$
\begin{aligned}
a_{N}\left(u_{N}, v_{N}\right)= & \int_{-1}^{1}\left(\frac{\partial^{2} u_{N}}{\partial x^{2}}(x, \cdot), \frac{\partial^{2} v_{N}}{\partial x^{2}}(x, \cdot)\right)_{N} d x \\
& +2\left\langle\frac{\partial^{4} u_{N}}{\partial x^{2} \partial y^{2}}, v_{N}\right\rangle_{N} \\
& +\int_{-1}^{1}\left(\frac{\partial^{2} u_{N}}{\partial y^{2}}(\cdot, y), \frac{\partial^{2} v_{N}}{\partial y^{2}}(\cdot, y)\right)_{N} d y
\end{aligned}
$$

The proof of the continuity and of the ellipticity of the form $a_{N}(\cdot, \cdot)$ requires two lemmas. The first can be found in [1, Lemma 2.3], the proof of the second is rather technical and is given in [2, Corollary V.2] in the more general case of weighted measures.

Lemma 3.2. The following inequality holds for any polynomial $\varphi_{N}$ in $\mathbf{P}_{N}^{0}(\Lambda)$ :

$$
\left\|\varphi_{N}^{\prime}\right\|_{0, \Lambda}^{2} \leq-\left(\varphi_{N}^{\prime \prime}, \varphi_{N}\right)_{N} \leq c\left\|\varphi_{N}^{\prime}\right\|_{0, \Lambda}^{2} .
$$

Lemma 3.3. The following inequality holds for any polynomial $\varphi_{N}$ in $\mathbf{P}_{N}^{00}(\Lambda)$ :

$$
c N^{-1}\left\|\varphi_{N}\right\|_{0, \Lambda}^{2} \leq\left(\varphi_{N}, \varphi_{N}\right)_{N} \leq c^{\prime}\left\|\varphi_{N}\right\|_{0, \Lambda}^{2} .
$$

We are now in a position to prove the following proposition.

Proposition 3.4. The form $a_{N}(\cdot, \cdot)$ satisfies the following properties of continuity:

(3.12) $\forall u_{N} \in \mathbf{P}_{N}^{00}(\Omega), \forall v_{N} \in \mathbf{P}_{N}^{00}(\Omega), \quad a_{N}\left(u_{N}, v_{N}\right) \leq c\left\|u_{N}\right\|_{2, \Omega}\left\|v_{N}\right\|_{2, \Omega}$, and of ellipticity

$$
\forall u_{N} \in \mathbf{P}_{N}^{00}(\Omega), \quad a_{N}\left(u_{N}, u_{N}\right) \geq c N^{-1}\left\|u_{N}\right\|_{2, \Omega}^{2} .
$$

Proof. Using (3.9) together with Lemma 3.3, we first deduce that

$$
\begin{aligned}
a_{N}\left(u_{N}, v_{N}\right) \leq & c\left\|\frac{\partial^{2} u_{N}}{\partial x^{2}}\right\|_{0, \Omega}\left\|\frac{\partial^{2} v_{N}}{\partial x^{2}}\right\|_{0, \Omega}+2\left\langle\frac{\partial^{4} u_{N}}{\partial x^{2} \partial y^{2}}, v_{N}\right\rangle_{N} \\
& +c\left\|\frac{\partial^{2} u_{N}}{\partial y^{2}}\right\|_{0, \Omega}\left\|\frac{\partial^{2} v_{N}}{\partial y^{2}}\right\|_{0, \Omega}
\end{aligned}
$$

and

$$
a_{N}\left(u_{N}, u_{N}\right) \geq c N^{-1}\left\|\frac{\partial^{2} u_{N}}{\partial x^{2}}\right\|_{0, \Omega}^{2}+2\left\langle\frac{\partial^{4} u_{N}}{\partial x^{2} \partial y^{2}}, u_{N}\right\rangle_{N}+c N^{-1}\left\|\frac{\partial^{2} u_{N}}{\partial y^{2}}\right\|_{0, \Omega}^{2}
$$


To estimate the middle term, we apply Lemma 3.2 in each direction, so that

$$
\left\langle\frac{\partial^{4} u_{N}}{\partial x^{2} \partial y^{2}}, v_{N}\right\rangle_{N} \leq c\left\|\frac{\partial^{2} u_{N}}{\partial x \partial y}\right\|_{0, \Omega}\left\|\frac{\partial^{2} v_{N}}{\partial x \partial y}\right\|_{0, \Omega}
$$

and similarly

$$
\left\langle\frac{\partial^{4} u_{N}}{\partial x^{2} \partial y^{2}}, u_{N}\right\rangle_{N} \geq c\left\|\frac{\partial^{2} u_{N}}{\partial x \partial y}\right\|_{0, \Omega}^{2} .
$$

Hence, we have proved on the one hand that

$$
a_{N}\left(u_{N}, v_{N}\right) \leq c\left\|u_{N}\right\|_{2, \Omega}\left\|v_{N}\right\|_{2, \Omega},
$$

which is the continuity property, and on the other hand that

$$
a_{N}\left(u_{N}, u_{N}\right) \geq c N^{-1}\left(\left\|\frac{\partial^{2} u_{N}}{\partial x^{2}}\right\|_{0, \Omega}^{2}+\left\|\frac{\partial^{2} u_{N}}{\partial x \partial y}\right\|_{0, \Omega}^{2}+\left\|\frac{\partial^{2} u_{N}}{\partial y^{2}}\right\|_{0, \Omega}^{2}\right),
$$

which is the desired ellipticity property.

The ellipticity property is not optimal, since the ellipticity constant is not independent of $N$. However, this result cannot be improved. Indeed, take the function

$$
u_{N}(x, y)=\varphi(x) \psi(y)
$$

where $\varphi$ is the polynomial $\left(1-\zeta^{2}\right)^{2} L_{N-1}^{\prime \prime \prime \prime}$ and where $\psi$ is any polynomial of $\mathbf{P}_{N-2}^{00}(\Lambda)$ satisfying

$$
\|\psi\|_{0, \Lambda} \leq c \quad \text { and } \quad|\psi|_{2, \Lambda} \geq c N^{4}
$$

It can be checked [1, Counterproposition 3.5] that

$$
a_{N}\left(u_{N}, u_{N}\right) \leq c N^{-1}\left\|u_{N}\right\|_{2, \Omega}^{2} .
$$

Note also that another collocation method is proposed in [8], where the collocation grid is built from the nodes of the usual Gauss-Lobatto formula; however a similar counterexample proves that, for this method, the constant of ellipticity is $\leq c N^{-2}$.

The first consequence of Proposition 3.4 is

Theorem 3.5. For any continuous function $f$ on $\Omega$, problem (3.3)-(3.5) has a unique solution $u_{N}$ in $\mathbf{P}_{N}^{00}(\Omega)$.

Another consequence is the abstract error estimate

$$
\left\|u-u_{N}\right\|_{2, \Omega}
$$

$$
\begin{array}{r}
\leq c N\left(\inf _{v_{N} \in \mathbf{P}_{N}^{00}(\Omega)}\left(\left\|u-v_{N}\right\|_{2, \Omega}+\sup _{w_{N} \in \mathbf{P}_{N}^{00}(\Omega)} \frac{\left(a-a_{N}\right)\left(v_{N}, w_{N}\right)}{\left\|w_{N}\right\|_{2, \Omega}}\right)\right. \\
\left.+\sup _{w_{N} \in \mathbf{P}_{N}^{00}(\Omega)} \frac{\left\langle f, w_{N}\right\rangle-\left\langle f, w_{N}\right\rangle_{N}}{\left\|w_{N}\right\|_{2, \Omega}}\right) .
\end{array}
$$

To estimate the terms on the right-hand side, we make use of properties (2.9) and (2.12). 
Theorem 3.6. Assume that the solution $u$ of problem (2.1)-(2.2) belongs to $H^{\sigma}(\Omega)$ for a real number $\sigma \geq 2$, and that the data $f$ are such that the function $\left(1-x^{2}\right)\left(1-y^{2}\right) f$ belongs to $H^{\rho}(\Omega)$ for a real number $\rho>1$. The following error estimate between $u$ and the solution $u_{N}$ of problem (3.3)-(3.5) holds:

$$
\left\|u-u_{N}\right\|_{2, \Omega} \leq c\left(N^{3-\sigma}\|u\|_{\sigma, \Omega}+N^{1-\rho}\left\|\left(1-x^{2}\right)\left(1-y^{2}\right) f\right\|_{\rho, \Omega}\right) .
$$

Proof. In (3.15), we choose $v_{N}$ equal to $\Pi_{N-3}^{2,0} u$, so that $\left(a-a_{N}\right)\left(u_{N}, v_{N}\right)$ is equal to 0 and, by (2.9),

$$
\left\|u-v_{N}\right\|_{2, \Omega} \leq c N^{2-\sigma}\|u\|_{\sigma, \Omega} .
$$

Moreover, using the exactness of the quadrature formula, we have for any $w_{N}$ in $\mathbf{P}_{N}^{00}(\Omega)$,

$$
\left\langle f, w_{N}\right\rangle-\left\langle f, w_{N}\right\rangle_{N}=\left\langle f-\mathcal{I}_{N-1}^{2} f, w_{N}\right\rangle .
$$

Recalling [13, Chapter 1, Theorem 11.3] that the mapping

$$
w \mapsto\left(1-x^{2}\right)^{-1}\left(1-y^{2}\right)^{-1} w
$$

is continuous from $H_{0}^{2}(\Omega)$ into $L^{2}(\Omega)$, we can write

$$
\left\langle f, w_{N}\right\rangle-\left\langle f, w_{N}\right\rangle_{N} \leq c\left\|\left(1-x^{2}\right)\left(1-y^{2}\right)\left(f-\mathcal{I}_{N-1}^{2} f\right)\right\|_{0, \Omega}\left\|w_{N}\right\|_{2, \Omega},
$$

whence, by virtue of (2.12),

$$
\left\langle f, w_{N}\right\rangle-\left\langle f, w_{N}\right\rangle_{N} \leq c N^{-\rho}\left\|\left(1-x^{2}\right)\left(1-y^{2}\right) f\right\|_{\rho, \Omega}\left\|w_{N}\right\|_{2, \Omega} .
$$

This completes the proof of the theorem.

From a numerical point of view, the $L^{2}(\Omega)$-norm of the error is also of great interest. The next proposition gives a better bound for this norm.

Proposition 3.7. If the assumptions of Theorem 3.6 are satisfied, the following error estimate between $u$ and the solution $u_{N}$ of problem (3.3)-(3.5) holds:

$$
\left\|u-u_{N}\right\|_{0, \Omega} \leq c\left(N^{1-\sigma}\|u\|_{\sigma, \Omega}+N^{-\rho}\left\|\left(1-x^{2}\right)\left(1-y^{2}\right) f\right\|_{\rho, \Omega}\right) .
$$

Proof. The proof relies on an Aubin-Nitsche duality argument, since we have

$$
\left\|u-u_{N}\right\|_{0, \Omega}=\sup _{g \in L^{2}(\Omega)} \frac{\left\langle u-u_{N}, g\right\rangle}{\|g\|_{0, \Omega}} .
$$

For any $g$ in $L^{2}(\Omega)$, we consider the solution $z$ of the problem

$$
\begin{cases}\Delta^{2} z=g & \text { in } \Omega, \\ z=\partial z / \partial n=0 & \text { on } \partial \Omega\end{cases}
$$

and we have the regularity estimate [10, Theorem 7.2.2.3] (see also the following remark)

$$
\|z\|_{4, \Omega} \leq c\|g\|_{0, \Omega}
$$

Next, we compute

$$
\begin{aligned}
\left\langle u-u_{N}, g\right\rangle=a\left(u-u_{N}, z\right)= & a\left(u-u_{N}, z-\Pi_{N-3}^{2,0} z\right) \\
& -\left\langle f, \Pi_{N-3}^{2,0} z\right\rangle+\left\langle f, \Pi_{N-3}^{2,0} z\right\rangle_{N},
\end{aligned}
$$


so that

$$
\begin{aligned}
\left\langle u-u_{N}, g\right\rangle \leq c( & \left\|u-u_{N}\right\|_{2, \Omega}\left\|z-\Pi_{N-3}^{2,0} z\right\|_{2, \Omega} \\
& +\left\|\left(1-x^{2}\right)\left(1-y^{2}\right)\left(f-\mathcal{F}_{N-1}^{2} f\right)\right\|_{0, \Omega} \\
& \left.\times\left(\sup _{z_{N} \in \mathbf{P}_{N}^{00}(\Omega)} \frac{\left\|\left(1-x^{2}\right)^{-1}\left(1-y^{2}\right)^{-1} z_{N}\right\|_{0, \Omega}}{\left\|z_{N}\right\|_{2, \Omega}}\right)\left\|\Pi_{N-3}^{2,0} z\right\|_{2, \Omega}\right) .
\end{aligned}
$$

Applying (3.16), (2.9), and (2.12), together with (3.18) and (3.19), gives the result.

Remark. By using the techniques of [10, Theorem 7.2.2.3], [4, §II], it can be checked that the mapping $f \mapsto u$, where $u$ is the solution of problem (2.1)(2.2), is continuous from $H^{s}(\Omega)$ into $H^{s+4}(\Omega)$ for any real number $s<s_{0}=$ $0.739 \ldots$. This proves the convergence of the solution of the discrete problem towards the exact one in the $H^{2}(\Omega)$-norm when $N$ tends to $+\infty$, whenever $f$ belongs to $H^{\rho}(\Omega), \rho>1$ : indeed, we have at least the estimates

$$
\begin{gathered}
\left\|u-u_{N}\right\|_{2, \Omega} \leq c N^{-\inf \{1.739, \rho-1\}}\|f\|_{\rho, \Omega}, \\
\left\|u-u_{N}\right\|_{0, \Omega} \leq c N^{-\inf \{3.739, \rho\}}\|f\|_{\rho, \Omega} .
\end{gathered}
$$

\section{ANOTHER Discretization}

Our conclusion in the previous section was that the collocation method is simple to formulate and very natural; however the lack of ellipticity of the discrete problem leads to nonoptimal error estimates. The aim of this last section is to propose another spectral technique to discretize problem (2.1)(2.2), which is no longer of collocation type but leads to optimal estimates in any dimension. As before, we first describe the discrete problem and then present its numerical analysis.

4.1. The discrete problem. The idea in constructing the discrete problem relies on the variational formulation (2.4) and consists in replacing the integrals which appear in this formulation by the quadrature formula (2.10) with $M$ equal to $N$ and $m$ equal to either 1 or 3 . For any sufficiently smooth functions $\varphi$ and $\psi$ on $\bar{\Lambda}$, we define the discrete product $(\varphi, \psi)_{N, m}$ by replacing $M$ by $N$ and $\Phi$ by the product $u v$ in the quadrature formula (2.10). To state the approximation of problem (2.1)-(2.2), we also need a discrete scalar product $\langle\cdot, \cdot\rangle_{N, m}$ on $\mathscr{C}^{2 m-2}(\bar{\Omega}) \times \mathscr{C}^{2 m-2}(\bar{\Omega})$, which we define by replacing each integral in the scalar product of $L^{2}(\Omega)$ by the quadrature formula (2.10). For functions $u$ and $v$ which both vanish at \pm 1 together with their first $(m-1) / 2$ derivatives, this can simply be written as:

$$
\langle u, v\rangle_{N, m}=\sum_{j=1}^{N-m} \sum_{k=1}^{N-m} u\left(\xi_{j}^{N, m}, \xi_{k}^{N, m}\right) v\left(\xi_{j}^{N, m}, \xi_{k}^{N, m}\right) \rho_{j}^{N, m} \rho_{k}^{N, m} .
$$

Next, we set

$$
a_{N, m}(u, v)=\langle\Delta u, \Delta v\rangle_{N, m} .
$$


For any continuous function $f$ on $\bar{\Omega}$, the discrete problem is written as follows:

$$
\begin{aligned}
& \text { find } u_{N} \text { in } \mathbf{P}_{N}^{00}(\Omega) \text { such that } \\
& \qquad \forall v_{N} \in \mathbf{P}_{N}^{00}(\Omega), \quad a_{N, m}\left(u_{N}, v_{N}\right)=\left\langle f, v_{N}\right\rangle_{N, m} .
\end{aligned}
$$

This discrete problem for $m=1$ has already been considered in [15], but with the zeros of $L_{N}^{\prime}$ replaced by the zeros of the derivative of the Chebyshev polynomial of degree $N$ and the $\rho_{j}^{N, 1}, 1 \leq j \leq N-1$, replaced by the corresponding Gauss-Lobatto Chebyshev weights, so that its numerical analysis requires a variational formulation of the continuous problem in weighted Sobolev spaces.

Remark. Problem (4.3) is no longer a collocation system. Indeed, in the case $m=1$, the number of interior grid points is no longer equal to the dimension of the space of test functions, i.e., it is equal to $(N-1)^{2}$ instead of $(N-3)^{2}$. In the case $m=3$, we are going to write the $(N-3)^{2}$ equations which are satisfied by the values of $u_{N}$ at the nodes $\left(\xi_{j}^{N, 3}, \xi_{k}^{N, 3}\right), 1 \leq j, k \leq N-3$. For this, we choose $v_{N}$ equal to the unique polynomial of $\mathbf{P}_{N}^{00}(\Omega)$ which vanishes in any point of the grid but not in $\left(\xi_{j}^{N, 3}, \xi_{k}^{N, 3}\right)$. This leads to the equations

$$
\begin{aligned}
\Delta^{2} u_{N}\left(\xi_{j}^{N, 3}, \xi_{k}^{N, 3}\right)= & f\left(\xi_{j}^{N, 3}, \xi_{k}^{N, 3}\right)-\lambda_{j}\left(\Delta^{2} u_{N}-f\right)\left(\xi_{j}^{N, 3}, \pm 1\right) \\
& -\lambda_{k}\left(\Delta^{2} u_{N}-f\right)\left( \pm 1, \xi_{k}^{N, 3}\right) \\
& -\lambda_{j} \lambda_{k}\left(\Delta^{2} u_{N}-f\right)( \pm 1, \pm 1),
\end{aligned}
$$

where the symbol \pm 1 stands for a summation on the value in -1 and the value in +1 . The $\lambda_{j}, 1 \leq j \leq N-3$, can be computed explicitly as functions of the $\rho_{j}^{N, 3}$ and $\xi_{j}^{N, 3}$. Hence, problem (4.3) can be interpreted as a "quasi-collocation" one. Note also that, in the case $m=3$, the associated mass matrix is diagonal, which allows for an efficient discretization algorithm for the corresponding time-dependent problem.

4.2. Numerical analysis. In view of the variational formulation of problem (4.3), the numerical analysis relies on the continuity and ellipticity of the form $a_{N, m}(\cdot, \cdot)$. As in $\S 3$, using the exactness of the quadrature formula and integrating by parts, we note that, for any polynomials $u_{N}$ and $v_{N}$ in $\mathbf{P}_{N}^{00}(\Omega)$,

$$
\begin{aligned}
a_{N, m}\left(u_{N}, v_{N}\right)= & \int_{-1}^{1}\left(\frac{\partial^{2} u_{N}}{\partial x^{2}}, \frac{\partial^{2} v_{N}}{\partial x^{2}}\right)_{N, m} d x \\
& +2 \int_{-1}^{1} \int_{-1}^{1}\left(\frac{\partial^{2} u_{N}}{\partial x \partial y}\right)\left(\frac{\partial^{2} v_{N}}{\partial x \partial y}\right) d x d y \\
& +\int_{-1}^{1}\left(\frac{\partial^{2} u_{N}}{\partial y^{2}}, \frac{\partial^{2} v_{N}}{\partial y^{2}}\right)_{N, m} d y .
\end{aligned}
$$

Thus, the properties of the form $a_{N, m}(\cdot, \cdot)$ are a straightforward consequence of the following lemma, which is well known in the case $m=1$ (see $[6, \S 3]$ ). Here, for the sake of completeness, we give a general proof for any odd value of the parameter $m$. 
Lemma 4.1. For odd values of a positive integer $m$, the following inequality holds for any polynomial $\varphi_{N}$ in $\mathbf{P}_{N}(\Lambda)$ :

$$
\left\|\varphi_{N}\right\|_{0, \Lambda}^{2} \leq\left(\varphi_{N}, \varphi_{N}\right)_{N, m} \leq c\left\|\varphi_{N}\right\|_{0, \Lambda}^{2} .
$$

Proof. Since the quadrature formula is exact on $\mathbf{P}_{2 N-1}(\Lambda)$, we see by using the expansion of $\varphi_{N}$ in the basis $\left(L_{n}\right)_{0 \leq n \leq N}$ that it suffices to prove the result for $\varphi_{N}=L_{N}$. Next, we note that the polynomial

$$
\psi_{N}=L_{N}^{2}-(-1)^{m} \frac{1}{N^{2}(N-1)^{2} \cdots(N-m+1)^{2}}\left(1-\zeta^{2}\right)^{m}\left(\frac{d^{m} L_{N}}{d \zeta^{m}}\right)^{2}
$$

is of degree $\leq 2 N-1$. Hence, applying the quadrature formula to $\psi_{N}$ and using its exactness, yields

$$
\begin{aligned}
\left(L_{N},\right. & \left.L_{N}\right)_{N, m}-\left\|L_{N}\right\|_{0, \Lambda}^{2} \\
= & (-1)^{m} \frac{1}{N^{2}(N-1)^{2} \cdots(N-m+1)^{2}} \\
& \times\left(\left(\frac{d^{m} L_{N}}{d \zeta^{m}},\left(1-\zeta^{2}\right)^{m} \frac{d^{m} L_{N}}{d \zeta^{m}}\right)_{N, m}-\left\|\left(1-\zeta^{2}\right)^{m / 2} \frac{d^{m} L_{N}}{d \zeta^{m}}\right\|_{0, \Lambda}^{2}\right) .
\end{aligned}
$$

Since the nodes of the quadrature formula are the zeros of $d^{m} L_{N} / d \zeta^{m}$, we obtain

$$
\begin{aligned}
\left(L_{N}, L_{N}\right)_{N, m}-\left\|L_{N}\right\|_{0, \Lambda}^{2}= & (-1)^{m+1} \frac{1}{N^{2}(N-1)^{2} \cdots(N-m+1)^{2}} \\
& \times\left\|\left(1-\zeta^{2}\right)^{m / 2} \frac{d^{m} L_{N}}{d \zeta^{m}}\right\|_{0, \Lambda}^{2} \cdot
\end{aligned}
$$

To compute the right-hand side, we check by induction on $m$ that

$$
\begin{aligned}
& \left(\frac{d^{m}}{d \zeta^{m}}\right)\left(\left(1-\zeta^{2}\right)^{m} \frac{d^{m} L_{n}}{d \zeta^{m}}\right) \\
& \quad=(-1)^{m}(n-m+1)(n-m+2) \cdots(n+m-1)(n+m) L_{n} .
\end{aligned}
$$

Integrating by parts gives

$$
\left(L_{N}, L_{N}\right)_{N, m}-\left\|L_{N}\right\|_{0, \Lambda}^{2}=(-1)^{m+1} \frac{(N+1)(N+2) \cdots(N+m)}{N(N-1) \cdots(N-m+1)}\left\|L_{N}\right\|_{0, \Lambda}^{2} .
$$

This proves the two inequalities of the lemma.

Using Lemma 4.1 in (4.5) gives at once

Proposition 4.2. The form $a_{N, m}(\cdot, \cdot)$ satisfies the following properties of continuity

$$
\forall u_{N} \in \mathbf{P}_{N}^{00}(\Omega), \quad \forall v_{N} \in \mathbf{P}_{N}^{00}(\Omega), \quad a_{N, m}\left(u_{N}, v_{N}\right) \leq c\left\|u_{N}\right\|_{2, \Omega}\left\|v_{N}\right\|_{2, \Omega}
$$

and of ellipticity

$$
\forall u_{N} \in \mathbf{P}_{N}^{00}(\Omega), \quad a_{N, m}\left(u_{N}, u_{N}\right) \geq c\left\|u_{N}\right\|_{2, \Omega}^{2} .
$$

Remark. The equation (4.8) explains why we do not use the quadrature formula with $m=2$ in our discretization. Indeed, if the same discrete problem were considered with $m=2$, the ellipticity constant would tend to 0 when $N$ tends to $+\infty$, so that the error estimates could not be optimal, in contrast to the following results.

The well-posedness result is now a straightforward consequence of Proposition 4.2 . 
Theorem 4.3. For any continuous function $f$ on $\bar{\Omega}$, problem (4.3) has a unique solution $u_{N}$ in $\mathbf{P}_{N}^{00}(\Omega)$.

We are now in a position to prove the error estimate.

Theorem 4.4. Assume that the solution $u$ of problem (2.1)-(2.2) belongs to $H^{\sigma}(\Omega)$ for a real number $\sigma \geq 2$, and that the data $f$ are such that the function $\left(1-x^{2}\right)\left(1-y^{2}\right) f$ belongs to $H^{\rho}(\Omega)$ for a real number $\rho>1$. The following error estimate between $u$ and the solution $u_{N}$ of problem (4.3) holds:

$$
\left\|u-u_{N}\right\|_{2, \Omega} \leq c\left(N^{2-\sigma}\|u\|_{\sigma, \Omega}+N^{-\rho}\left\|\left(1-x^{2}\right)\left(1-y^{2}\right) f\right\|_{\rho, \Omega}\right) .
$$

Proof. Exactly as in $\S 3$, we have

$$
\begin{array}{r}
\left\|u-u_{N}\right\|_{2, \Omega} \leq c\left(\inf _{v_{N} \in \mathbf{P}_{N}^{00}(\Omega)}\left(\left\|u-v_{N}\right\|_{2, \Omega}+\sup _{w_{N} \in \mathbf{P}_{N}^{\mathbf{0} 0}(\Omega)} \frac{\left(a-a_{N, m}\right)\left(v_{N}, w_{N}\right)}{\left\|w_{N}\right\|_{2, \Omega}}\right)\right. \\
\left.+\sup _{w_{N} \in \mathbf{P}_{N}^{\mathbf{0} 0}(\Omega)} \frac{\left\langle f, w_{N}\right\rangle-\left\langle f, w_{N}\right\rangle_{N, m}}{\left\|w_{N}\right\|_{2, \Omega}}\right) .
\end{array}
$$

Choosing $v_{N}=\Pi_{N-1}^{2,0} u$ cancels the second term and gives the estimate

$$
\left\|u-v_{N}\right\|_{2, \Omega} \leq c N^{2-\sigma}\|u\|_{\sigma, \Omega} .
$$

To estimate the last term, we use the exactness of the quadrature formula on $\mathbf{P}_{2 N-1}(\Lambda)$ together with the fact that $\mathscr{J}_{N}^{m} f$ belongs to $\mathbf{P}_{N-2}(\Lambda)$ to deduce that, for any $w_{N}$ in $\mathbf{P}_{N}^{00}(\Omega)$,

$$
\left\langle f, w_{N}\right\rangle-\left\langle f, w_{N}\right\rangle_{N, m}=\left\langle f-\mathscr{I}_{N}^{m} f, w_{N}\right\rangle .
$$

Next, we recall that the mapping $w \mapsto\left(1-x^{2}\right)^{-1}\left(1-y^{2}\right)^{-1} w$ is continuous from $H_{0}^{2}(\Omega)$ into $L^{2}(\Omega)$, so that the use of (2.12) gives

$$
\left\langle f, w_{N}\right\rangle-\left\langle f, w_{N}\right\rangle_{N, m} \leq c N^{-\rho}\left\|\left(1-x^{2}\right)\left(1-y^{2}\right) f\right\|_{\rho, \Omega}\left\|w_{N}\right\|_{2, \Omega} .
$$

The estimate (4.11) now follows from (4.12) to (4.14).

The arguments for proving the next result are exactly the same as for Proposition 3.7.

Proposition 4.5. If the assumptions of Theorem 4.4 are satisfied, the following error estimate between $u$ and the solution $u_{N}$ of problem (4.3) holds:

$$
\left\|u-u_{N}\right\|_{0, \Omega} \leq c\left(N^{-\sigma}\|u\|_{\sigma, \Omega}+N^{-\rho}\left\|\left(1-x^{2}\right)\left(1-y^{2}\right) f\right\|_{\rho, \Omega}\right) .
$$

\section{CONCLUding REMARKS}

We propose two methods for a model fourth-order problem with Dirichlet boundary conditions. Both of them can be easily extended to the threedimensional case (see [1]). The first $(\S 3)$ is of collocation type, hence it is very natural, but the error estimates cannot be optimal: one order of accuracy is lost in the square and two in the cube. The second method ( $\$ 4)$ relies on the variational formulation of the problem and leads to optimal error estimates, in both cases $m=1$ and $m=3$. The two choices for $m$ differ in the fact that the number of boundary terms in the discrete problem increases with $m$, at the 
expense of the number of interior nodes. Numerical simulation (in progress) may decide what choice is better among these two optimal methods.

The previous analysis is extended in [1] to inhomogeneous boundary conditions, i.e., when conditions (2.2) are replaced by

$$
u=g_{J} \quad \text { and } \quad \frac{\partial u}{\partial n_{J}}=h_{J} \quad \text { on } \Gamma_{J}, J=1,2,3,4 .
$$

Indeed, assume that the functions $g_{J}$ (resp. $\left.h_{J}\right), J=1,2,3,4$, belong to $H^{3 / 2}\left(\Gamma_{J}\right)$ (resp. $H^{1 / 2}\left(\Gamma_{J}\right)$ ), that they are continuous on $\bar{\Gamma}_{J}$ and differentiable in $\mathbf{a}_{J-1}$ and $\mathbf{a}_{J}$, and that the following compatibility conditions hold:

$$
\begin{array}{ll}
g_{J}\left(\mathbf{a}_{J}\right)=g_{J+1}\left(\mathbf{a}_{J}\right), & \frac{d g_{J}}{d \tau_{J}}\left(\mathbf{a}_{J}\right)=h_{J+1}\left(\mathbf{a}_{J}\right), \\
h_{J}\left(\mathbf{a}_{J}\right)=-\frac{d g_{J+1}}{d \tau_{J+1}}\left(\mathbf{a}_{J}\right), & \frac{d h_{J}}{d \tau_{J}}\left(\mathbf{a}_{J}\right)=-\frac{d h_{J+1}}{d \tau_{J+1}}\left(\mathbf{a}_{J}\right),
\end{array} \quad J=1,2,3,4 .
$$

Then the discrete problem is set up as follows: find $u_{N}$ in $\mathbf{P}_{N}(\Omega)$ satisfying (3.3) (resp. the variational equation in (4.3)) together with the boundary equations

$$
\begin{aligned}
& u_{N}(\mathbf{x})=g_{J}(\mathbf{x}) \quad \text { and } \quad \frac{\partial u_{N}}{\partial n_{J}}(\mathbf{x})=h_{J}(\mathbf{x}), \quad \mathbf{x} \in \Xi_{N J}, J=1,2,3,4, \\
& u_{N}\left(\mathbf{a}_{J}\right)=g_{J}\left(\mathbf{a}_{J}\right), \quad \\
& \frac{\partial u_{N}}{\partial n_{J+1}}\left(\mathbf{a}_{J}\right)=h_{J+1}\left(\mathbf{a}_{J}\right), \quad \frac{\partial_{N} u_{N}}{\left.\partial n_{J} \partial \mathbf{a}_{J+1}\right)=h_{J}\left(\mathbf{a}_{J}\right)}\left(\mathbf{a}_{J}\right)=\frac{d h_{J}}{d \tau_{J}}\left(\mathbf{a}_{J}\right),
\end{aligned}
$$

It is proven in [1, $\S 3$ and 4$]$ that this problem has a unique solution and the error estimates of Theorems 3.6 and 4.4 are still valid in this case.

\section{BIBLIOGRAPHY}

1. C. Bernardi, G. Coppoletta, and Y. Maday, Some spectral approximations of multidimensional fourth-order problems, Internal Report 90021, Laboratoire d'Analyse Numérique, Université Pierre et Marie Curie, Paris, 1990.

2. C. Bernardi and Y. Maday, Some spectral approximations of one-dimensional fourth-order problems, Progress in Approximation Theory (P. Nevai and A. Pinkus, eds.), Academic Press, Boston, 1991, pp. 43-116.

3. __ Spectral methods, Handbook of Numerical Analysis (P. G. Ciarlet and J.-L. Lions, eds.), North-Holland (to appear).

4. C. Bernardi and G. Raugel, Méthodes d'éléments finis mixtes pour les équations de Stokes et de Navier-Stokes dans un polygone non convexe, Calcolo 18 (1981), 255-291.

5. C. Canuto, M. Y. Hussaini, A. Quarteroni, and T. A. Zang, Spectral methods in fluid dynamics, Springer-Verlag, New York, 1987.

6. C. Canuto and A. Quarteroni, Approximation results for orthogonal polynomials in Sobolev spaces, Math. Comp. 38 (1982), 67-86.

7. P. J. Davis and P. Rabinowitz, Methods of numerical integration, 2nd ed., Academic Press, Orlando, 1984.

8. D. Funaro and W. Heinrichs, Some results about the pseudospectral approximation of onedimensional fourth-order problems, Numer. Math. 58 (1990), 399-418.

9. L. Gatteschi, Su una formula di quadratura "quasi gaussiana". Tabulazione delle ascisse d'integrazione et delle relative costanti di Christoffel, Atti Accad. Sci. Torino Cl. Sci. Fis. Mat. Natur. 98 (1963-64), 641-661. 
10. P. Grisvard, Elliptic problems in nonsmooth domains, Pitman, Boston, 1985.

11. E. B. Karpilovskaya, $A$ method of collocation for integro-differential equations with biharmonic principal part, U.S.S.R. Comput. Math. and Math. Phys. 10 (1970), no. 6, 240-246.

12. O. Koutchmy (in preparation).

13. J.-L. Lions and E. Magenes, Problèmes aux limites non homogènes et applications, Vols. 1 and 2, Dunod, Paris, 1968.

14. Y. Maday, Résultats d'approximation optimaux pour les opérateurs d'interpolation polynômiale, C. R. Acad. Sci. Paris Sér. I. 312 (1991), 705-710.

15. Y. Maday and B. Métivet, Chebyshev spectral approximation of Navier-Stokes equations in a two-dimensional domain, Modél. Math. Anal. Numér. 21 (1987), 93-123.

16. S. A. Orszag, Accurate solution of the Orr-Sommerfeld stability equation, J. Fluid Mech. 50 (1971), 689-703.

Analyse Numérique, C.N.R.S. ANd Université Pierre et Marie Curie, Tour 55-65, 5 ìme étage, 4 place Jussieu, F-75252 Paris Cedex 05, France

E-mail address, C. Bernardi: bernardi@frcirp81.bitnet

E-mail address, Y. Maday: maday@frcirp81.bitnet 